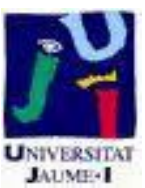

Título artículo / Títol article: Ecological momentary assessment for chronic pain in fibromyalgia using a smartphone: A randomized crossover study

Autores / Autors

A. Garcia-Palacios, R. Herrero, M.A. Belmonte, D. Castilla ,J. Guixeres, G. Molinari, R.M. Baños, C. Botella

Revista:

European Journal of Pain, 18(6)

Versión / Versió:

Post-print autor

Cita bibliográfica / Cita

GARCIA- PALACIOS, A., et al. Ecological momentary bibliogràfica (ISO 690): assessment for chronic pain in fibromyalgia using a smartphone: a randomized crossover study. European Journal of Pain, 2014, vol. 18, no 6, p. 862-872.

url Repositori UJI:

http://hdl.handle.net/10234/127286 
Running head: Comparing EMA using smartphone vs. paper

\title{
Ecological Momentary Assessment for chronic pain in fibromyalgia using a smartphone: A randomized crossover study
}

\author{
A. Garcia-Palacios ${ }^{1,2}$, R. Herrero ${ }^{1}$, M.A. Belmonte ${ }^{3}$, D. Castilla ${ }^{1}$, J. Guixeres $^{4}$, G. \\ Molinari $^{1}$, R. M. Baños ${ }^{5,2} \&$ C. Botella ${ }^{1,2}$ \\ ${ }^{1}$ Universitat Jaume I, Dpt. Psicología Básica, Clínica y Psicobiología. Spain. \\ ${ }^{2}$ Ciber Fisiopatología Obesidad y Nutrición (CB06/03 Instituto Salud Carlos III, Spain). \\ ${ }^{3}$ Hospital General de Castellón. Servicio de Reumatología, Spain \\ ${ }^{4}$ Labhuman. Universidad Politécnica de Valencia, Spain. \\ ${ }^{5}$ Universidad de Valencia, Dept. Personalidad, Evaluación y Tratamientos Psicológicos, \\ Spain.
}

Category: Original Article

\section{Funding Sources}

The research presented in this paper was funded in part by Fundació La Marató de TV3 (Ajuts de la Marató de TV3 2006), Plan de Promocion de la investigacion Universitat Jaume I, (Fundacio Caixa Castello-Bancaixa, P11B2009-30) and by Generalitat Valenciana, Redes de Excelencia ISIC (ISIC/2012/012).

\section{Conflicts of interest}

None declared

\section{'What's already known about this topic?'}

- The use of Ecological Momentary Assessment (EMA) and Real time Data Capture (RTDC) in the field of chronic pain is a very useful tool for clinicians and researchers because it makes possible to gather more accurate and complete ratings of relevant variables.

\section{What does this study add?}

- The findings of this study contribute with data supporting the use of smartphones for RTDC in a sample of fibromyalgia patients with an important proportion of participants with low educational levels and low familiarity with technology

\section{Corresponding author:}

Guadalupe Molinari

Universitat Jaume I. Dpt. Psicologia Basica, Clinica y Psicobiologia

Avda Vicent Sos Baynat s/n 12071 Castellon. Spain

Phone: +34 964387645

Fax: +34 964729267

Email: molinari@uji.es 


\begin{abstract}
Background: Daily diaries are a useful way of measuring fluctuations in pain-related symptoms. Although this approach entails a repeated assessment, traditional diaries do not assure the gathering of data in real-time, and therefore do not solve the problem of retrospective assessment. Ecological momentary assessment (EMA) and, more concretely, real-time data capture (RTDC) by means of electronic diaries can help to improve repeated assessment in chronic pain.
\end{abstract}

Method: The present work contributes to this line of research by comparing the accuracy and acceptability of an RTDC method running on a smartphone using a crossover design for a sample with a low level of education and low familiarity with technology. Forty-seven women diagnosed with fibromyalgia were randomly assigned to one of two conditions: 1) paper diary - smartphone diary, and 2) smartphone diary paper diary, using each assessment method for one week.

Results: The findings of this study showed that the smartphone diary made it possible to gather more accurate and complete ratings of relevant variables. Besides, this method was well accepted by a sample of fibromyalgia patients referred by a public hospital with a large proportion of participants with low level of education and low familiarity with technology.

Conclusion: The findings of this study support the use of smartphones for ecological momentary assessment in the field of chronic pain. These methods could help clinicians and researchers to gather more accurate ratings of relevant pain-related variables even in populations with low educational levels and low familiarity with technology. 


\section{Introduction}

Chronic pain is a complex experience that requires a multidimensional perspective (Flor and Turk, 2011). Patients experience pain-related symptoms every day and a useful way of measuring the fluctuations of the symptoms and their relationship with other variables is by using a daily diary. This method includes fixed-interval assessments, usually employing a retrospective perspective. Shiffman, Stone, and Rufford (2008) include them as a special case in the category of Ecological Momentary Assessment (EMA), defined as "methods using repeated collection of real-time data on subjects' behavior and experience in their natural environment" (p. 3) (Shiffman et al., 2008). Daily diaries gather repeated data but not in real-time and therefore do not solve the problem of retrospective assessment.

Retrospective assessment tends to produce higher estimations of events. The symptoms tend to be described as more frequent, more intense, and longer lasting (Broderick et al., 2008; Gwaltney et al., 2008). Some studies also found that retrospective measures are highly context dependent (Fredrickson, 2000; Stone and Broderick, 2007). Also, low compliance rates have been found when comparing reported and actual compliance with paper diaries (Stone et al., 2003). These limitations have an effect on the reliability of these measures (Fredrickson, 2000). Information and communication technologies (ICTs) can help to improve traditional methods of assessment through real-time data capture (RTDC) by making it easier for patients to answer questions during programmed sessions with the help of computers or electronic devices.

Bolger, Davis and Rafaeli (2003) suggested that RTDC is particularly useful for examining fluctuations of phenomena in real-time and for obtaining measures of 
change over time. RTDC also improves adherence and acceptability compared to traditional methods (Morris et al., 2010; Wilhelm \& Schoebi, 2007)and allows patients to receive feedback in real-time (Beasly et al., 2008).

Stone and Broderick (2007) found a correlation of around 0.75 between recalled pain and average momentary pain for a one-week period and confirmed that retrospective reports of pain are higher when compared with the average of RTDC data for the same period. These authors also reviewed data confirming that RTDC is yielding important findings like the high degree of variability within a day and across days in the levels of pain and the relationship between pain and other variables like mood or activity. As this promising field progresses, Shiffman et al. (2008) indicate some concerns regarding special populations, , or environmental or personal factors that could limit the use of RTDC. One population to consider is people unfamiliar with or fearful of technology.

This research aims to contribute to this line of research by comparing the accuracy and acceptability of an RTDC assessment method running on a smartphone in a single-centre, randomized, crossover study with fibromyalgia sufferers. The specific aims were: 1) to compare the accuracy of two EMA methods (paper diary and smartphone diary); 2) to explore the relationship between aggregated EMA data and retrospective data; and 3) to explore the acceptability of the two EMA procedures in a population with low level of education and low familiarity with technology.

\section{Methods}

\section{Participants}


Figure 1 shows a flow chart diagram including the recruitment process. The exclusion criteria for this study were suffering a severe mental illness or severe sensory impairments (visual, motor, or hearing).

\section{-Insert Figure 1-}

Seventy-four participants were screened and a total of 47 women participated in the study; their ages ranged from 37 to $65(M=48.05 ; S D=7.95)$. All participants were volunteers and were recruited from Castellon General Hospital in Spain. All participants met the criteria for fibromyalgia syndrome according to the American College of Rheumatology (Wolfe et al., 1990) and were diagnosed by a rheumatologist.

With respect to the educational profile, $10 \%$ had not finished elementary education (less than 8 years of education), $47.5 \%$ had elementary education, $25 \%$ had high school education, and only $17.5 \%$ had a university degree.

Regarding familiarity with technology, $17.5 \%$ of participants had no experience with computers, $37.5 \%$ had used computers just a few times (less than 10 times), and $45 \%$ usually used computers. With regard to Internet use, $27.5 \%$ had never used the Internet, $27.5 \%$ had used it a few times (less than 10 times), and $45 \%$ used it at least twice a week. In reference to the use of mobile phones, $100 \%$ of the sample used mobile phones, but $22.5 \%$ of the sample did not know how to read SMS and $37.5 \%$ did not know how to write an SMS. Also, $32.5 \%$ of the sample had used a device with a touch screen at least once; the rest had never used a device with a touch screen.

\section{Measures}

Three types of measures were included in this study:

1) EMA measures. We included the recording of three key variables in the study of pain: pain intensity, fatigue intensity, and mood. Pain and fatigue were rated on 0 to 10 Numerical Rating Scales (NRS) from "no pain/fatigue" to "worst pain/fatigue 
you can imagine". Mood was assessed with a face-based pictorial seven-point scale. A time-based approach with fixed intervals was chosen for this study. Participants were asked to complete these three ratings three times a day.

These measures were analysed following two principles: the presence or lack of presence of the rating and the compliance with the time frame in which the participant had to fill out the records. In this sense and according to Stone et al. (2003) a record completed outside the specified time range was treated as one that was not completed within $+/-30$ minutes of the exact time. According to this rule, records were classified into four categories: a) complete record: a record completed in the stipulated time; b) complete record, out of time: a record completed outside of the time range; c) incomplete record: a record not totally completed, with at least one piece of data missing; d) totally incomplete record: a totally missed record.

2) Weekly measures of pain and fatigue. Patients completed the Brief Pain Inventory (BPI) (Cleeland and Ryan, 1994; Badia et al., 2003) and the Brief Fatigue Inventory (BFI) (Mendoza et al., 1999) once a week in order to gather a retrospective rating of average pain/fatigue intensity. The scales from these inventories asking for average pain/fatigue intensity in the last week were the ones chosen for this study.

3) Self-report inventories to assess the two EMA conditions. These measures were designed for this study. The first was a questionnaire to evaluate each condition separately (acceptability questionnaire) which consisted of fourteen items with a range of responses from 1 "totally agree" to 5 "totally disagree". This questionnaire was administered by the assessor, who requested the opinion of the participant about different relevant areas regarding acceptability. The second measure was a comparison questionnaire developed in order to discover the participants' preferences among the two conditions (preferences questionnaire). This questionnaire was administered by the 
assessor once the study ended and consisted of nine items with three different options of response "traditional", "mobile device", or "indistinct". The items included in these inventories are displayed in Tables 3 and 4.

\section{Materials}

Two different modalities of the assessment procedures were developed: one using a mobile device and the other using a paper-and-pencil traditional diary.

Our research team developed a software application (F-EMA) running on a mobile device. The hardware used was a Smartphone HTC Diamond 1 with the following specifications: $51 \times 102 \times 11.5 \mathrm{~mm}$; ROM $4352 \mathrm{MB}$; RAM $192 \mathrm{MB} ; 480 \times$ 640 display resolution; 2.8 " display diagonal; 16 bit/pixel display colour depth; audio stereo sound. The software was run on Windows Mobile 6.1. In Figure 2 we offer a picture of the mobile application. The reason for using a mobile phone was that the idea was to develop an application that could be used by patients in their natural environments.

The assessment was carried out three times a day. The default schedule was set as $9 \mathrm{am}, 3 \mathrm{pm}$, and $9 \mathrm{pm}$. The system allowed these times to be adjusted according to the particular needs of each participant. An audio signal indicated that the participant should fill out the rating scales. If the user did not complete them, the audio signal sounded again every minute during the first 15 minutes and then every 15 minutes during the following hour. After that time the application considered that the user was not able to answer and the assessment was not performed. The application included the option of not only seeing the images of the scales but also listening to audio-recorded instructions, which were included with the intention of making the system easier to use for a wider number of people (for example elderly people or people with some visual impairment). 
Usability studies were carried out. Results showed that F-EMA was an easy tool to use and to learn to use (Castilla et al., 2012)

A traditional pencil-and-paper diary was also designed including the same scales as those on the mobile device (see Figure 3). The only difference between the two conditions was that the mobile device automatically recorded the time at which the participant answered, while in the traditional self-record the participants had to fill in the time of the day at which they completed the rating.

-Insert Figures 2 and 3-

\section{Procedure}

This is a single-centre, randomized, crossover study with fibromyalgia sufferers. Participants were recruited from the rheumatology unit of Castellon General Hospital in Spain. Approval was granted by the Institutional Review Board. All participants attended voluntarily and signed an informed consent form. The participants completed a brief interview, where information about demographics and their clinical status was gathered, as well as a technological profile.

After the initial assessment, which comprised a one-hour session, participants were randomly assigned to one of two conditions: 1) P: paper-and-pencil diary smartphone diary; 2) S: smartphone diary - paper-and pencil diary (see Figure 4).

At the start of the first week participants attended an individual information session (S1) in which the experimenter provided the corresponding self-record (paper vs. smartphone) and provided verbal instructions about the use of the self-record. Experimenters explained to the participants that they were going to be asked to assess three important aspects in the field of chronic pain: pain intensity, fatigue intensity, and mood. The experimenter explained the meaning of the scales and the way in which they should be rated. Participants had to fill in the self-record three times a day every day, so 
the experimenters asked for three different hours during the day (morning, afternoon, and evening) that were convenient in the daily schedule of each participant. The participants practised rating the scales with the experimenter, and finally an information sheet with the meaning of each scale and instructions for filling in the record was given to each participant. Participants recorded the assessments daily in their natural environments for seven days.

At the end of the first week a second session was held (S2). In this session experimenters received the self-record data from the participants, administered the Acceptability Questionnaire regarding the self-record procedure used during the week (paper or smartphone), and performed a weekly rating of pain and fatigue (average pain intensity and fatigue measured by the BPI and BFI). Then each participant received the other self-record (paper or smartphone). The experimenters explained the procedure and the participants practised the method of rating the scales and were given an information sheet with the instructions. They recorded the assessments daily in their natural environments for seven more days.

At the end of the second week, a third session was held (S3). In this session experimenters received the self-record diaries and administered the Acceptability Questionnaire regarding the self-record procedure used that week and the Preference Questionnaire in order to compare both conditions. Participants also gave weekly ratings of average fatigue and pain intensity using the scales included in the BPI and BFI.

-Insert Figure 4-

\section{Results}

Accuracy of two EMA methods: smartphone versus paper and pencil 
The first objective of this study was to compare the accuracy of a traditional paper-andpencil diary with a smartphone diary. T-tests for related samples were conducted in order to compare the adherence of participants to the instructions in both conditions, using the four categories set out.

The results showed significant differences in three of the four categories (see Table 1). Significant differences were obtained in complete records, showing a higher number of complete records in the smartphone condition than in the traditional condition with a high effect size. A significant difference was found between the two conditions regarding incomplete records, showing a higher number of incomplete records in the traditional condition with a high effect size. Significant differences were also obtained regarding the totally incomplete records, showing a higher number of totally incomplete records in the smartphone condition, although the effect size here was moderate. Finally, no significant differences were found regarding records completed out of time.

We would like to highlight that, taking into account that the total number of records was 21 per week (three per day for seven days), the rate of complete records was much higher with the use of the mobile device (18.2: 86.66\%) compared with the use of the traditional diary (11.12: 52.95\%).

\section{-Insert Table 1 -}

We also conducted correlations between aggregated data of the paper diary and of the smartphone. Regarding pain intensity, $r=0.79, p<.001$, and regarding fatigue, $r$ $=0.88, p<0.001$. In both cases the correlations were positive and statistically significant.

\section{Relationship between aggregated EMA data and retrospective data}


Another of our objectives was to compare recall-based and real-time data. To achieve this goal we compared aggregated EMA data with the retrospective rating of pain intensity and pain fatigue that the participants reported once a week during the study. The mean ratings and standard deviation are reported in Table 2. T-tests revealed significant differences between the recall-based ratings and the EMA data in both pain and fatigue intensity. Aggregated EMA data using the traditional diary were lower than the retrospective ratings regarding pain intensity and fatigue intensity. Aggregated EMA data using the smartphone were also lower than the recall-based ratings regarding pain intensity and fatigue intensity. That is, participants tended to describe their symptoms as more intense when they gave retrospective weekly ratings.

We also examined the correlation between aggregated EMA and recall-based data for pain and fatigue intensity. Positive and significant correlations were found between aggregated EMA data and recall-based data regarding pain rated with the paper diary and the weekly average pain intensity $(r=0.59 ; p<0.001)$ and with the smartphone diary and the weekly average pain intensity $(r=0.39 ; p<0.02)$. With regard to fatigue, correlations were calculated for fatigue intensity rated with the paper diary and the weekly measure $(r=0.67 ; \mathrm{p}<0.001)$ and fatigue intensity rated with the smartphone and the weekly measure of fatigue intensity $(r=0.47 ; p<0.01)$. In all cases correlations were positive and statistically significant.

-Insert Table 2-

\section{Acceptability of the two EMA procedures}

Our final aim was to explore the acceptability of the EMA methods (paper-and pencil vs. smartphone). In order to analyse the acceptability, satisfaction, and preference between the two conditions, $\mathrm{t}$-tests for related samples were conducted, comparing the answers to each item of the Acceptability Questionnaire and the Preference 
Questionnaire. Regarding the Acceptability Questionnaire, the results showed significant differences in six items (see Table 3): the smartphone condition was perceived as easier to use and faster to answer. The participants perceived the instructions of the smartphone condition significantly easier to follow than those of the paper condition, even when in both conditions the instructions were exactly the same. Regarding the general opinion of the participants about the two conditions, the smartphone method was evaluated as significantly easier and more useful than the paper method. Finally, significant differences in opinions were found regarding whether other people with the same condition should use the assessment procedures, showing that the smartphone was more highly recommended by the participants than the paper-andpencil diary.

\section{-Insert Table 3-}

In Table 4 we show the percentage of participants reporting a preference for one of the two methods (paper vs. smartphone) in several domains. The results showed that participants preferred the smartphone method in general (65\% vs. 15\%); a higher percentage of them also thought it was possible to answer faster with the smartphone (70\% vs. $17.5 \%)$ and that the smartphone method was more useful (50\% vs. 5\%). The smartphone was also considered easier to remember to fill in (90\% vs. $2.5 \%)$, more comfortable to complete (55\% vs. $2.5 \%$ ), and more comfortable to carry ( $85 \%$ vs. 2.5\%). A higher number of participants also reported that the paper diary bothered them more $(45 \%$ vs. $10 \%)$.

-Insert Table 4- 


\section{Discussion}

Ecological Momentary Assessment (EMA) and more concretely Real-Time Data Capture (RTDC) methods are being tested in the field of chronic pain and the results indicate that these assessment procedures can improve the accuracy and validity of the traditional assessment methods.

This work is a contribution to this field of research. Our team developed a RTDC procedure running on a smartphone. After the design phase, our main aim was to compare the use of this RTDC with a traditional paper-and-pencil diary.

Using a randomized crossover design, participants used each method for a period of one week. The correlations between the paper and smartphone data were very high, meaning that the two methods were measuring very similar variables. That is, as expected, the use of a smartphone did not produce important changes in the measure. However, when comparing the frequency of complete and incomplete records, the mobile device condition showed higher levels of compliance than the traditional condition, presenting a higher rate of complete records $(86.66 \%$ vs. $52.95 \%)$, and significantly lower number of incomplete records. Daily diaries and other EMA methods were introduced in order to obtain ratings of key variables in real-time and in natural environments, thus reducing the bias of retrospective data. The fact that the person does not complete the record on time could have a negative influence on the validity of the data. It is possible that other variables are contaminating the measurement, distorting and biasing the information gathered. These biases invalidate the benefits of EMA methods. Therefore, we consider on-time completion of the ratings to be an important variable. The results confirm that the smartphone diary obtained a higher compliance. We would like to point out that, although we were able to have control over the actual compliance with the smartphone because it recorded the ratings 
in real-time, we were not able to have control over the actual compliance of the paper diary and we had to rely on the reported compliance. Participants were given a paper diary and were instructed to fill it in three times a day. We did not have a way of knowing whether they filled in the ratings at the specified times or whether they filled the diary forward or backward (they only included the time when they filled in each rating in the diary, but there was no way of knowing whether it was the real time). Stone et al. (2003) included a method of discovering the actual compliance with the paper diary by using a mechanism that could detect when the diary was opened and closed. They found that while reported compliance was $90 \%$, actual compliance was only $11 \%$. Our study was not so focused on compliance, we were more interested in exploring the utility of a smartphone for RTDC and because of that we compared it to what we think is the most common and standard procedure: a paper diary like the one currently used in regular practice. It is important to notice that, even considering reported compliance, it was significantly lower with the paper diary than with the smartphone.

A second objective was to explore the relationship between aggregated EMA data and retrospective data. We found positive and significant correlations between aggregated EMA data (with both methods) and recall-based data, indicating that both procedures were measuring a similar variable. On the other hand, and as expected, retrospective reports of pain and fatigue were higher than aggregated EMA data. Participants tended to describe their symptoms as more intense when they gave retrospective weekly ratings. Our results are in line with those found in the RTDC literature reviewed by Stone and Broderick (2007) who found a correlation between recalled pain and average momentary pain for a one-week period of around 0.75 and higher pain retrospective reports when compared with the average of RTDC data for the 
same period. In our case we explored not only pain but also another important painrelated symptom, fatigue. The same findings were made in relation to this symptom.

Human memory is limited in capacity and time of retention. In the case of pain, retrospective assessment tends to produce higher estimations of events. The symptoms tend to be described as more frequent, more intense, and longer lasting (Houtveen and Oei, 2007; Broderick et al., 2008). This could be due to the two rules of "peak pain" and "closest pain", whereby more weight is given to the peak of pain experienced and to the most recently experienced pain, rather than equal weight being given to each experience (Redelmeier and Kahneman, 1996). We believe this has important implications for the experience of pain. This finding means that patients remember feeling more pain than they actually felt when looking at the daily ratings. Thus there is a distorted view of the intensity of the pain and other related symptoms. On one hand, this supports the role of cognitive factors in the experience of pain such as catastrophizing (Keefe et al., 2004). On the other hand the comparison between real-time data and recall data could be used as a therapeutic tool to promote decatastrophizing.

The last objective was to explore the acceptability, satisfaction, and preferences regarding the two assessment methods. Several studies indicate that e-diaries have higher acceptability than traditional assessment methods (Cranford et al., 2006; Wilhelm and Schoebi, 2007). Other studies report that traditional diaries present the advantage of being more familiar to users and maybe easier to use (Stone et al., 2003). Another variable to take into account is the specific population to be assessed. In our case, we know that fibromyalgia is more prevalent in people between 40 and 60 years old (Baldry, 2001) and with lower educational and socio-economic levels (Mas et al,, 2008). These features are usually related with less familiarity with technology. Because of that we consider it important to explore the acceptability of the smartphone diary and 
the paper diary in this specific population. The results obtained show that the smartphone diary was rated as easier, more useful, and more highly recommended than the paper diary. Regarding preferences, most participants preferred the smartphone over the paper diary. In summary, the smartphone presented a higher acceptability than the traditional method, even in a sample with a large proportion of participants with low familiarity with technology and low educational level. The evolution of technology is one of the biggest achievements of our recent history; however, it is important to make this technology available to everybody who can benefit from it. Specific populations could be at risk of not benefiting from technological innovations because of the digital divide, and we believe researchers and clinicians have a responsibility to offer technological tools that have been submitted to a process of careful evaluation in order to reach most people who could benefit from them. This has been our goal in the development of the tool we present in this study.

This study presents some limitations. We already indicated that we could not report actual compliance with the paper diary and we had to rely on reported compliance. In any case, compliance was much better with the smartphone than with the paper diary. Another limitation is that the sample size could be higher. The strength of the sample was that it was representative of the population that suffers from fibromyalgia in our country given that the participants were referred by the rheumatology unit of a public hospital. Also, the effect sizes obtained in the statistical analysis when differences were found were satisfactory. A final limitation is that the application developed runs only on smartphone platforms using Windows Mobile software, leaving out other important mobile platforms. It is also true that the software developed is very simple and easy to program. In fact, we are now developing an updated version that could run on the main smartphone platforms. 
This study and others in the same line belong to a promising line of research that is providing interesting data for the field of pain but is still developing. More studies evaluating the use of smartphones for EMA methods are needed. Another future issue in this line of research is the use of these procedures in longitudinal studies in order to analyse the relationship between different variables and individual differences in the experience of pain. Finally, fast advances in technology will reduce costs and improve the feasibility of using patients' mobile phones for EMA and for giving therapeutic feedback during the administration of an intervention.

The findings of this study contribute with data supporting the use of smartphones for RTDC in the field of chronic pain. This is a very useful tool for clinicians and researchers because it makes it possible to gather more accurate and complete ratings of relevant variables. Besides the good results regarding data collection, this method was accepted well by a sample of fibromyalgia patients with an important proportion of participants with low educational levels and low familiarity with technology referred by the rheumatology unit of a public hospital.

\section{Author Contribution}

All authors have contributed to the manuscript. Azucena Garcia-Palacios and Cristina Botella contributed to the conception and design of the study. Diana Castilla and Jaime Guixeres conducted the design and development of the computer software. The statistical analysis and the interpretation of data were conducted by Rocio Herrero and Azucena Garcia-Palacios. An initial draft of the manuscript was witten by Rocio Herrero and Guadalupe Molinari, under the guidance of Azucena García Palacios. Rosa Baños provided helpful advice revising it critically. All authors contributed to and approved the final draft. 


\section{References}

Badia, X., Muriel, C., Gracia, A., Nunez-Olarte, J.M., Perulero, N., Galvez, R., Carulla, J., Cleeland, C.S. (2003).Validation of the Spanish version of the Brief Pain Inventory in patients with oncological pain [in Spanish]. Med Clin (Barc) 120,52-59.

Baldry, P.E. (2001). Clinical characteristics and biopathophysiological mechanisms of fibromyalgia syndrome. In: Myofascial pain and fibromyalgia syndromes, P.E. Baldry,ed. (Edinburgh: Churchill Livingstone), pp. 351-377.

Beasly, J., Riley, W., Davis, A., Singh, J. (2008). Evaluation of a PDA-based dietary assessment and intervention program: a randomized controlled trial. J Am Coll Nutr 27, 280-286.

Bolger, N., Davis, A., Rafaeli, E. (2003). Diary methods: capturing life as it is lived. Ann Rev Psychol 54,579-616.

Broderick, J.E., Schwartz, J.E., Vikingstad, G., Pribbernow, M., Grossman, S., Stone, A.A. (2008). The accuracy of pain and fatigue items across different reporting periods. Pain 139,146-157.

Castilla, D., Botella, C., Garcia-Palacios, A., Farfallini, L., Miralles, I. (2012). Experience-sampling methodology with a mobile device in fibromyalgia. Int J Telem Appl 2012, ID 162673. doi:10.1155/2012/162673

Cleeland, C.S., Ryan, K.M. (1994). Pain assessment: global use of the brief pain inventory. Ann Acad Med 23,129-38.

Cranford, N.J., Shrout, P., Iida, M., Rafaeli, E., Yip, T., Bolger, N. (2006). A procedure for evaluating sensitivity to Within-Person Change: Can mood measures in diary studies detect change reliability? Pers soc psychol bull 32, 917-929.

Cohen, J. (1988). Statistical power analysis for the behavioral sciences, 2nd ed. (Hillsdale: Lawrence Earlbaum Associates). 
Flor, H., Turk, D.C. (2011). Assessment of characteristics of pain and pain behaviors: laboratory and Clinical Methods. In: Chronic pain. An integrated biobehavioral approach, Flor H. Flor and D.C. Turk, eds. (Seattle: IASP Press) pp. 217-252.

Fredrickson, B. L. (2000). Extracting meaning from past affective experiences: The importance of peaks, ends, and specific emotions. Cogn Emotion 14, 577-606.

Gwaltney, C.J., Shields, A.L., Shiffman, S. (2008). Equivalence of electronic and paperand-pencil administration of patient-reported outcome measures: A meta-analytic review. Val Health 11,322-333.

Gendreau, M., Hufford, M.R., Stone, A.A. (2003). Measuring clinical pain in chronic widespread pain: selected methodological issues. Best Pract Res Clin Rheumatol $17,575-592$.

Houtveen, J.H., Oei, N.Y.L. (2007). Recall bias in reporting medically unexplained symptoms comes from semantic memory. J Psychosom Res 62, 277-282.

Keefe, F.J., Rumble, M.E., Scipio, C.D., Giodano, L.A., Perri, L.M. (2004). Psychological aspects of persistent pain: current state of the science. J Pain 5,195-211. Mas, A.J., Carmona, L., Valverde, M., Rivas, B., EPISER Study group. (2008). Prevalence and impact of fibromyalgia on function and quality of life in individuals from the general population: results from a nationwide stud in Spain. Clin Exp Rheumatol 26,519-526.

Mendoza, T.R., Wang, X.S., Cleeland, C.S., Morrissey, M., Johnson, B.A., Wendt, J.K., Huber, S.L. (1999). The rapid assessment of fatigue severity in cancer patients: use of the Brief Fatigue Inventory. Cancer 85,1186-1196.

Morris, M.E., Kathawala, Q., Leen, T.K., Gorenstein, E.E., Guilak, F., Labhard, M., Dellew, W. (2010). Mobile therapy: case study evaluations of a cell phone application for emotional self-awareness. J Med Internet Res 12, 10. 
Redelmeier, D., Kahneman, D. (1996). Patients' memories of painful medical treatments: real-time and retrospective evaluations of two minimally invasive procedures. Pain 66,3-8.

Shiffman, S., Stone, A.A., Hufford, M. (2008). Ecological momentary assessment. Ann Rev Clin Psychol 4,1-32.

Stone, A.A., Broderick, J.E. (2007). Real-time data collection for pain: appraisal and current status. Pain Med 8,85-93.

Stone, A.A., Shiffman, S., Schwartz, J.E., Broderick, J.E., Hufford, M.R. (2003). Patient compliance with paper and electronic diaries. Control Clin Trials 24,182-199. Wilhelm, P., Schoebi, D. (2007). Assessing mood in daily life-Structural validity, sensitivity to change, and reliability o a short-scale to measure three basic dimensions of mood. Eur J Psychol Assessm 23,258-267.

Wolfe, F., Smythe, H.A., Yunos, M.B., Bennett, R.M., Bombardier, C., Goldenberg, D.L. (1990). The American College of Rheumatology 1990 criteria for the classification of fibromyalgia: report of the Multicenter Criteria Committee. Arthritis Rheum 33,16072. 
Figure 1. Study Flow Diagram

Figure 2. Appearance of RTDC application

Figure 3. Appearance of paper-and-pencil diary

Figure 4. Procedure Schema 